\title{
Converting existing service to fully automatic operation
}

\author{
F. Fabbian \\ MTR Corporation Limited, Hong Kong
}

\begin{abstract}
Fully Automatic Operation (FAO) is rapidly becoming the system of choice for Mass Rapid Transit Systems. The technology is mature and there is now over 20 years experience in this technology. Existing systems would also benefit by being automated. The challenges of converting an existing manned system to FAO are more challenging than a green field installation. The MTR Corporation of Hong Kong is currently operating one line which is FAO and has a plan to convert the remaining lines. This is a system that transports $2.6 \mathrm{M}$ per day and trains that carry up to 2,700 passengers. This paper will share the planning strategy and business case for this conversion - the Hong Kong story.

Keywords: fully automatic operation.
\end{abstract}

\section{Introduction}

This paper will describe the automatic operation developments undertaken in MTRCL including the automatic turnaround function at terminus, automatic train door opening and closing, fully automatic operation for the new Disneyland Resort Line and the studies undertaken for the conversion of the existing operation to fully automatic operation.

MTRCL has adopted a progressive approach to the development of fully automatic operation. The automatic turnaround function at terminal stations was introduced in 1999 which was followed by the implementation of automatic train door opening and closing functions for the existing fleet and the fully automatic line, Disneyland Resort Line.

Feasibility studies were conducted to review the possible technical and operational impact and opportunities for converting the existing operation into fully automatic operation. These included a review of the operational 
requirements, development of functional requirements for fully automated operation, reliability and risk assessments, external peer review of the technical specifications, and an energy study on potential savings after converting to fully automatic operation.

\section{Automatic turnaround}

Before the modification for automatic turnaround operation in 1999, the turnaround operation at a terminus was manned by two train operators, one at each end of the train. This was to allow the train to be driven to the designated turnaround track by one operator at the front or arrival cab who would then shutdown the cab so that the rear or departure cab at the other end could be started by another train operator for its return journey

To support the automatic turnaround function, both the trackside and trainborne ATC equipment were modified to support inter-cab communication through trackside telemetry and automatic train control authority transfer from the arrival cab to the departure cab thereby replacing the manual operation. With the modifications, the whole turnaround operation would simply be initiated by the train operator at the arrival cab. The train operator would then walk to the other end of the train to continue the journey in the opposite direction. Thus, the total manpower requirement was reduced.

In addition to staff cost saving, the automatic turnaround project provided the additional benefit of service consistency of train operation. It also provided the opportunity for MTR to review the operation requirements, method of train failure handling and operational rules and procedures when the system is under automatic turnaround operation. It was identified that new operational procedures were required to handle the following scenarios:-

(a) Broken rail within the turnaround areas: the operational rules would now require the first trip at the beginning of daily operation must be manned by operators to visually check the rail condition;

(b) Passenger trespassing at terminal station with no platform screen door (PSD): the operational rules would now require for non-PSD terminal station that the departure driving cab must be manned when the train is entering the terminal station platform.

\section{Automatic train door opening and closing}

A new circuit was built to interface with train door control circuits to enable train doors to open automatically when trains stopped at platforms. The new control circuit was built by programmable logic control (PLC) which would check the traction control circuits, zero speed detection relays and mode selector position to ensure train doors would only open at the correct side when a train was stopped correctly at the platform stopping point. A dwell time saving of $1.5 \mathrm{~s}$ was achieved with this new function. A service trial was conducted with all trains converted in early 2005. 
The new control circuit was subsequently expanded with the additional interface of digital voice PA to achieve the automatic train door closing function. A lookup table of preset dwell time of each platform was stored in the PLC. The PLC would start counting down the preset dwell time when a train is stopped at a platform. The digital voice PA would be triggered when the count down of $10 \mathrm{~s}$ is reached. The automatic train doors closing command would be issued when the preset dwell time is reached. A service trial is underway with 13 trains converted to monitor any service related impact.

\section{Disneyland Resort Line}

MTRCL awarded a signalling contract in 2002 for the seventh operational line Disneyland Resort Line (DRL) for the Hong Kong Disney Theme Park. This new line which was opened in August 2005 was designed for fully automated operation with two stations and two 4-car trains.

The signalling system of DRL employs the ALCATEL's SelTrac40 Communication Based Train Control (CBTC) solution, which was designed for automatic operation. The ATP system of SelTrac40 ensures safety by maintaining the minimum train separation of one fixed virtual block. All block lengths were calculated in accordance with civil engineering speed limitations and train characteristics. The ATP system also provides the following features to safeguard the train movement:-

(a) Rollback protection - The ATP safeguards the train movement in the assigned direction of travel by detecting the route set, operation mode and other trainborne inputs. If a backward movement over $1 \mathrm{~m}$ is detected by the ATP, emergency brake is activated.

(b) Overspeed protection - The ATP limits the speed of trains according to safe operating and civil engineering speed limits in all driving modes. The Emergency brake will be activated by the ATP whenever the train speed is above the ATP limit.

(c) Door interlocking - The ATP interlocks with the train door control circuit to ensure the correct side of train doors can only be opened at the designated position in the platform when the train speed is zero and the service brake is applied.

The ATO system of DRL regulates the train speed using a combination of feedback and open-loop control algorithms to achieve and maintain the target speed imposed by the ATP system and supported train movement within the passenger ride quality criteria, i.e. maximum jerk limit of $0.2 \mathrm{~m} / \mathrm{s}^{3}$ for all interstation runs. The ATO system provides for the programmed station stops including train door opening and closing according to the station dwell time control as well as on-board audio announcement control and cab display. The ATO also supports the following non-vital functions:- 
(a) Remote control from ATS - To support driverless operation, ATO interfaces with other trainborne sub-systems to respond upon receiving remote commands from ATS. These commands included emergency brake reset, train hold, train door open/close command, service speed adjustment, VOBC switchover, pantograph control, reset to rolling stock traction or auxiliary power circuits, etc.

(b) Status Reporting to ATS - ATO makes use of the wireless LAN to report to the ATS continuously the current train status, e.g. train ID, operation mode, emergency brake status, train speed, door status, train hold status, travel direction, station stopping status and rolling stock alarms.

(c) Energy saving in peak and non-peak hours - ATO adjusts train speed according to the ATS regulation strategy based on the energy saving strategy for peak and non-peak hours. By adjusting the dwell time and travel time under different regulation strategies, the train can be driven at the optimum speed to minimise the energy consumption at different periods of a day.

(d) Audio announcement control - ATO automatically generates announcement requests to trigger audio messages at pre-defined train locations along the path of the train.

Without track circuits, the train detection was achieved by wayside tag, trainborne tachometer updating and continuous communication between OnBoard Control Unit (OBCU) and Wayside Control Unit (WCU) through a wireless network. To boost the system reliability, the CBTC system made use of the dual redundant OBCU's from both ends of the train to support the features of rear-end automatic operation and remote speed restriction movement, which would allow a train to move forward to retain localization when the OBCU's at both ends lost the localization information.

To support maintenance works by locomotives within the new line, the system was customised to allow non-ALCATEL equipped trains to move safely within DRL and interface with ALSTOM's signalling system in Tung Chung Line (TCL) to ensure a smooth and safe cross line operation. This was achieved with the introduction of axle counters at fixed locations along DRL thereby creating a traditional fixed block operation for route setting when a nonALCATEL train is running inside DRL area.

The ALCATEL wayside equipment was extended and overlaid on the existing ALSTOM wayside area so that the DRL train could be operated between the DRL area and the depot in Siu Ho Wan of TCL. The new signalling system was designed to protect DRL trains and provide all the ATP functions in Coded Manual operation mode using track occupancy, point position and signal status information from the ALSTOM system.

This inter-operability design experience provided useful invaluable inputs in overcoming similar challenges of conversion in future. 


\section{Studies for fully automatic operation}

In April, 2004, a working group was formed to look into the different aspects of converting the existing train fleet (106 trains of 8-car consists) to support fully automatic operation.

The benefits identified for adopting automatic operation included improved regulation of train service, saving in manpower and energy. Moreover, automatic operation would enable the train service regulation to be more responsive to customer demand especially during festive occasions and peak hours. This improved responsiveness would also accelerate service recovery after perturbations. Automatic operation would also improve system reliability by removing variable human errors, mostly caused by monotonous work. Experience from other railway operations indicated train service could be more reliable than other lines with train operators who would be redeployed to other value added tasks of better customer service.

The working group analyzed the existing railway systems, including rolling stock, signalling, communication and other infrastructure, to determine the gaps for a fully automated operation railway. To support a fully automated operation railway, our systems must be retrofitted to cope with the following functional requirements.

(a) Automatic train door control to support platform duty according to pre-defined platform dwell

(b) Automatic train starting when ATP allows the train to depart after a series of automated checks and confirmation sequence are performed.

(c) Automatic jogging movement when a train stops within a limited distance away from the designated stopping point.

(d) Two-way communication between Operation Control Centre (OCC) and passengers inside saloon.

(e) Remote monitoring of train status by operators in OCC so that operator can visualize the health status of the trainborne equipment remotely.

(f) Remote control from OCC to trainborne equipment, e.g. fault reset to ATC \& auxiliary equipment, pantograph control, train initialization checking, etc.

(g) Upgrade the existing radio network to support the real-time two-way communication between trainborne equipment and OCC.

Train modification would be required on train door control and provisions of interfaces for automatic operation to support jogging movement, remote control and fault reset. A highly reliable radio system is required for maintaining communication to passengers during normal and emergency situations and the radio system is essential for remote control and fault resetting functions.

To mitigate platform edge hazards such as passengers falling on track, platform screen doors or intrusion detection systems would be required at all stations. The market suppliers were invited to comment on the proposed requirements and the system that they may offer. 


\section{Reduced power consumption}

Apart from the long-term saving of the re-deployment of train operator staff, another major saving is traction energy. A study to quantify the possible energy saving of automatic operation was carried out.

The study included an in-depth review of our system configuration including the track layout scheme, electrical infrastructure and the characteristics of the train fleet, and service pattern (e.g. timetable and passenger activities on platform). A traction energy consumption model of the existing system configuration was built. Based on this model, the expected energy saving was simulated for different operation scenarios.

The simulated result concluded that since automatic operation could effectively minimize the turnaround time at terminals, the saved time could be used to extend the inter-station run time and thereby reducing the energy consumption. Extended run time meant that trains could be run at a lower speed to save energy. Another major contribution of the shortened turnaround time was the reduction of the number of trains required for the same service requirement. Trains may also be out-stabled at sidings hence avoiding the energy cost of transferring them to and from the depot.

\section{Service performance target}

One of the key objectives of the working group is to define the appropriate service performance targets of automatic operation.

Instead of using the performance target from other railways which may not be fully applicable to MTRCL, we have adopted a bottom-up approach in projecting the expected performance based on the existing system performance with the expected new functions.

For instance, there is currently little service delay arising from a minor overrun or stop short at a platform stop since the train operator can respond immediately to resolve the situation. However, under automatic operation, this incident will probably cause a service delay if no automated corrective measure was introduced. To address this issue, a new functional requirement of automatic train jogging is required when the system detects a minor overrun or stop short.

After completing this exercise to review all incident scenarios, it was concluded that improved system reliability must be achieved in order to maintain the same current service performance. This review also assists in defining the necessary requirements of automatic operation as well as the necessary operational procedures.

\section{Human resource and community issues}

A major challenge with implementing fully automatic operation on an existing railway is to have the staff appreciate the company's vision for service enhancement and ambition to meet customer expectation. A well planned HR strategy and communications will have to account for the affected 
staff numbers, run-down by natural attrition i.e. retirement or departure, and staff deployment to new extensions. There will also be the opportunity to multi-skill and redevelop skills of some staff to handle other responsibilities in the railway system. For example, more staff can be put on the network to enable a more flexible and responsive system to meet the needs of customers when they are travelling in the system.

The successful application of fully automatic operation in the Hong Kong Airport People Mover, and recently the MTRCL's Disneyland Resort Line has rendered fully automatic operation gain wide acceptance by legislators, politicians, public and MTRCL's own staff. More extensive implementation in the existing railway lines will be complemented by a PR plan to manage community issues e.g. safety, loss of jobs. The PR plan will cover study visits to other railways running on fully automatic operation for the key players such as legislators, regulators and politicians.

\section{Conclusion}

MTRCL has taken a progressive approach to the introduction of automatic operation, i.e. automatic turnaround, automatic train door opening / closing and Disneyland Resort Line which is fully automatic.

Based on the experience obtained, it is concluded that the technology for automatic operation is mature with a proven track record. This technology would provide a good means to match the service supply to demand both economically and commercially. 